\title{
Some Theorems Concerning Pseudo-Random Numbers
}

\author{
By D. L. Jagerman
}

Some quantitative theorems concerning the use of pseudo-random numbers will be presented. Let $x_{1}, \cdots, x_{P}$ be a given sequence satisfying

$$
0 \leqq x_{j} \leqq 1, \quad 1 \leqq j \leqq P,
$$

and $f(x)$ a real, integrable function; then the first four theorems estimate the quantity

$$
\left|\frac{1}{P} \sum_{j=1}^{P} f\left(x_{j}\right)-\int_{0}^{1} f(x) d x\right| .
$$

Further restrictions on the sequence $x_{j}$ will be imposed through a trigonometric sum. Let $n \neq 0$ be an integer, and let

$$
e(x)=e^{i 2 \pi x}
$$

then effective estimates for the size of

$$
\left|\sum_{j=1}^{p} e\left(n x_{j}\right)\right|
$$

will be required. Restrictions on the function $f(x)$ will be imposed by means of its Fourier coefficients. Thus, let $f(x)$ be given by

$$
f(x)=\int_{0}^{1} f(u) d u+\sum_{n=-\infty}^{\infty} c_{n} e(n x),
$$

in which the prime shows the absence of the term $n=0$; then growth restrictions on $c_{n}$ will be required. The fifth theorem is concerned with multiply sequences; it provides an estimate for the deviation of such a sequence from the ideal uniformly distributed case. The symbols $[x]$ and $\{x\}$ will be used to denote the integral part and the fractional part of $x$, respectively.

Theorem 1. $C>0, K>0, \alpha>0, \beta<1, \nu>1+\alpha \ni$

$$
\begin{aligned}
&\left|c_{n}\right| \leqq C|n|^{-\nu}, \quad\left|\sum_{j=1}^{P} e\left(n x_{j}\right)\right|<K|n|^{\alpha} P^{\beta} \\
& \Rightarrow\left|\frac{1}{P} \sum_{j=1}^{P} f\left(x_{j}\right)-\int_{0}^{1} f(x) d x\right|<\frac{2 K C(\nu-\alpha)}{\nu-\alpha-1} P^{\beta-1} .
\end{aligned}
$$

Proof. The Fourier series for $f(x)$ is

$$
f(x)=\sum_{n=-\infty}^{\infty} c_{n} e(n x)
$$

Received December 28, 1964. 
in which

$$
c_{n}=\int_{0}^{1} e(-n x) f(x) d x
$$

Thus, since the sequence $x_{j}$ obeys the inequality $0 \leqq x_{j} \leqq 1$, one has

$$
\frac{1}{P} \sum_{j=1}^{p} f\left(x_{j}\right)-\int_{0}^{1} f(x) d x=\sum_{n=-\infty}^{\infty} c_{n} \frac{1}{P} \sum_{j=1}^{P} e\left(n x_{j}\right) .
$$

Since $\left|c_{n}\right|$ and $\left|(1 / P) \sum_{j=1}^{P} e\left(n x_{j}\right)\right|$ are even functions of $n,(8)$ may be put in the form

$$
\left|\frac{1}{P} \sum_{j=1}^{P} f\left(x_{j}\right)-\int_{0}^{1} f(x) d x\right| \leqq 2 \sum_{n=1}^{\infty}\left|c_{n}\right|\left|\frac{1}{P} \sum_{j=1}^{P} e\left(n x_{j}\right)\right| . \quad \vee
$$

The conditions of the theorem imply

$$
\left|\frac{1}{P} \sum_{j=1}^{P} f\left(x_{j}\right)-\int_{0}^{1} f(x) d x\right| \leqq 2 K C P^{\beta-1} \sum_{n=1}^{\infty} \frac{1}{n^{\nu-\alpha}} .
$$

Since

$$
\sum_{n=1}^{\infty} \frac{1}{n^{\nu-\alpha}}<1+\int_{1}^{\infty} \frac{d x}{x^{\nu-\alpha}}=\frac{\nu-\alpha}{\nu-\alpha-1}
$$

the theorem follows.

Theorem 2. $C>0, K>0, \alpha>0, \beta<1, \nu=1+\alpha \ni$

$$
\begin{aligned}
\left|c_{n}\right| \leqq C|n|^{-\nu}, \quad\left|\sum_{j=1}^{P} e\left(n x_{j}\right)\right| \leqq K|n|^{\alpha} P^{\beta}, \quad P \geqq 3^{\alpha /(1-\beta)} \\
\Rightarrow\left|\frac{1}{P} \sum_{j=1}^{P} f\left(x_{j}\right)-\int_{0}^{1} f(x) d x\right|<\frac{2 C}{\alpha} P^{\beta-1}\left[2 K(1-\beta) \ln P+\left(\frac{3}{2}\right)^{\alpha}\right] .
\end{aligned}
$$

Proof. The proof is the same as for Theorem 1 up to (9). One has

$$
\left|\frac{1}{P} \sum_{j=1}^{P} f\left(x_{j}\right)-\int_{0}^{1} f(x) d x\right| \leqq 2 K C P^{\beta-1} \sum_{1 \leqq n \leqq P^{(1-\beta) / \alpha}} \frac{1}{n^{\nu-\alpha}}+2 C \sum_{n>P^{(1-\beta) / \alpha}} \frac{1}{n^{\nu}},
$$

in which the estimate

$$
\left|\frac{1}{P} \sum_{j=1}^{P} e\left(n x_{j}\right)\right| \leqq 1
$$

was used in the second sum. Since

$$
\sum_{n>P^{(1-\beta) / \alpha}} \frac{1}{n^{\nu}}<\int_{\left[P^{(1-\beta) / \alpha}\right]}^{\infty} \frac{d x}{x^{\nu}}=\frac{1}{(\nu-1)\left[P^{(1-\beta) / \alpha}\right]^{\nu-1}},
$$

and

$$
\left[P^{(1-\beta) / \alpha}\right]>P^{(1-\beta) / \alpha}-1=P^{(1-\beta) / \alpha}\left(1-P^{-(1-\beta) / \alpha}\right) \geqq \frac{2}{3} P^{(1-\beta) / \alpha},
$$

one has

$$
\sum_{n>P^{(1-\beta) / \alpha}} \frac{1}{n^{\nu}}<\frac{1}{\nu-1}\left(\frac{3}{2}\right)^{\nu-1} P^{-(1-\beta)(\nu-1) / \alpha},
$$


and, hence,

$$
2 C \sum_{n>P(1-\beta) / \alpha} \frac{1}{n^{\nu}}<\frac{2 C}{\alpha}\left(\frac{3}{2}\right)^{\alpha} P^{\beta-1}
$$

Also, since

$$
\sum_{1 \leqq n \leqq P(1-\beta) / \alpha} \frac{1}{n^{\nu-\alpha}}<1+\int_{1}^{P(1-\beta) / \alpha} \frac{d x}{x}=1+\frac{1-\beta}{\alpha} \ln P<2 \frac{1-\beta}{\alpha} \ln P,
$$

substituting (17) and (18) into (12) establishes the theorem.

Theorem 3. $C>0, K>0, \alpha>0, \beta<1,1<\nu<1+\alpha \ni$

$$
\begin{gathered}
\left|c_{n}\right| \leqq C|n|^{-\nu}, \quad\left|\sum_{j=1}^{P} e\left(n x_{j}\right)\right| \leqq K|n|^{\alpha} P^{\beta}, \quad P \geqq 3^{\alpha /(1-\beta)} \\
\Rightarrow\left|\frac{1}{P} \sum_{j=1}^{P} f\left(x_{j}\right)-\int_{0}^{1} f(x) d x\right|<2 C P^{-(1-\beta)(\nu-1) / \alpha}\left[\frac{K}{1-\nu+\alpha}+\frac{1}{\nu-1}\left(\frac{3}{2}\right)^{\nu-1}\right] .
\end{gathered}
$$

Proof. The proof of this theorem is the same as that of Theorem 2 up to (16). One has

$$
\sum_{1 \leqq n \leqq P(1-\beta) / \alpha} \frac{1}{n^{\nu-\alpha}}<\int_{0}^{P(1-\beta) / \alpha} \frac{d x}{x^{\nu-\alpha}}=\frac{1}{1-\nu+\alpha} P^{(1-\beta)(1-\nu+\alpha) / \alpha}
$$

and, hence, substituting (16) and (19) into (12) establishes the theorem.

Theorem 4. $C>0, K>0, \alpha>0, \beta<1, \nu>1, \nu<\alpha \ni$

$$
\begin{aligned}
\left|c_{n}\right| \leqq C|n|^{-\nu}, \quad & \left|\sum_{j=1}^{P} e\left(n x_{j}\right)\right| \leqq K|n|^{\alpha} P^{\beta}, \quad P \geqq 3^{\alpha /(1-\beta)} \\
& \Rightarrow\left|\frac{1}{P} \sum_{j=1}^{P} f\left(x_{j}\right)-\int_{0}^{1} f(x) d x\right| \\
& <2 C P^{-(1-\beta)(\nu-1) / \alpha}\left[\frac{K}{1-\nu+\alpha}\left(\frac{4}{3}\right)^{1-\nu+\alpha}+\frac{1}{\nu-1}\left(\frac{3}{2}\right)^{\nu-1}\right] .
\end{aligned}
$$

Proof. The proof of this theorem is the same as that of Theorem 2 up to (16). One has

$$
\begin{aligned}
\sum_{1 \leqq n \leqq P^{(1-\beta) / \alpha}} \frac{1}{n^{\nu-\alpha}}<\int_{1}^{P(1-\beta) / \alpha} \frac{d x}{x^{\nu-\alpha}} & <\frac{\left(P^{(1-\beta) / \alpha}+1\right)^{1-\nu+\alpha}}{1-\nu+\alpha} \\
& \leqq \frac{1}{1-\nu+\alpha}\left(\frac{4}{3}\right)^{1-\nu+\alpha} P^{(1-\beta)(1-\nu+\alpha) / \alpha} .
\end{aligned}
$$

Substituting (16) and (20) into (12) establishes the theorem.

A multiply sequence is defined by the recurrence relation

$$
x_{j+1}=\left\{\lambda x_{j}+\frac{\mu}{m}\right\}, \quad j \geqq 0,
$$

in which $\lambda>1, m>0, \mu \geqq 0$ are integers, and $x_{0}$ is arbitrarily chosen. Franklin [1] showed that, for almost all $x_{0}$, a multiply sequence is equidistributed. Let $m x_{0}$ be an integer which is relatively prime to $m$, then $m x_{j}$ is the sequence of integers 
customarily employed as pseudo-random numbers. Inductively, one easily establishes the following explicit representation:

$$
x_{j}=\left\{\lambda^{j} x_{0}+\frac{\mu}{m} \frac{\lambda^{j}-1}{\lambda-1}\right\} .
$$

If $m x_{0}$ is an integer, then the sequence $x_{j}$ is clearly periodic in the sense that $x_{j+m}=x_{j}$ for all $j$; however, it is generally desirable that the sequence contain as many members which are incongruent modulo $m$ as possible. This is accomplished by choosing for $\lambda$ a primitive root modulo $m$. A statistical function of interest is the distribution function, $\omega(\alpha)$. Let $P$ denote the period of the sequence; then, if $T(\alpha)$ is the number of elements of the sequence which do not exceed $\alpha$,

$$
\omega(\alpha)=\frac{T(\alpha)}{P} .
$$

Since $\omega(\alpha)=0$ for $\alpha \leqq 0$ and $\omega(\alpha)=1$ for $\alpha \geqq 1$, it is convenient to restrict $\alpha$ so that $0<\alpha<1$. The following discontinuous function will aid in the determination of $\omega(\alpha)$. Let

$$
\begin{array}{ll}
H_{\alpha}(x)=1, & 0 \leqq x<\alpha, \\
H_{\alpha}(x)=0, & \alpha \leqq x<1,
\end{array}
$$

and define $H_{\alpha}(x)$ for all $x$ by periodic extension; then

$$
\omega(\alpha)=\frac{1}{P} \sum_{j=1}^{P} H_{\alpha}\left(x_{j}\right) .
$$

The special case $\mu=0$ of (22) will be studied in which $\lambda$ is a primitive root modulo $m$ and $P=\phi(m)$, where $\phi(m)$ is the totient. Thus

$$
x_{j}=\left\{\lambda^{j} x_{0}\right\},
$$

and $m x_{0}$ is one of the numbers of a reduced residue system modulo $m$. In order to investigate the distribution function of this sequence, several lemmas are needed. Let

$$
\rho(x)=\frac{1}{2}-\{x\}
$$

then:

Lemma 1.

$$
|\omega(\alpha)-\alpha| \leqq\left|\frac{1}{P} \sum_{j=1}^{P} \rho\left(x_{j}\right)\right|+\left|\frac{1}{P} \sum_{j=1}^{P} \rho\left(x_{j}-\alpha\right)\right|, \quad 0<\alpha<1 .
$$

Proof. One has

$$
H_{\alpha}(x)=\alpha+\rho(x)-\rho(x-\alpha),
$$

which may be established by consideration of the two cases $0 \leqq x<\alpha$ and $\alpha \leqq x<1$. Thus, from (25),

$$
\omega(\alpha)=\alpha+\frac{1}{P} \sum_{j=1}^{P} \rho\left(x_{j}\right)-\frac{1}{P} \sum_{j=1}^{P} \rho\left(x_{j}-\alpha\right) .
$$


The lemma follows from (29).

LemMa 2.

$$
t>0 \Rightarrow-\frac{1}{2 t}+t \int_{-1 / t}^{0} \rho(x+u) d u \leqq \rho(x) \leqq \frac{1}{2 t}+t \int_{0}^{1 / t} \rho(x+u) d u .
$$

Proof. From the monotonicity of $[x]$, one has

$$
t \int_{-1 / t}^{0}[x+u] d u \leqq[x] \leqq t \int_{0}^{1 / t}[x+u] d u
$$

Since

$$
[x]=x-\frac{1}{2}+\rho(x),
$$

substitution of this into (30) yields the lemma.

LEMMA 3. $t>0$

$$
\begin{aligned}
& \Rightarrow-\frac{1}{2 t}+\sum_{n=-\infty}^{\infty} d_{n} e(n x) \leqq \rho(x) \leqq \sum_{n=-\infty}^{\infty} c_{n} e(n x)+\frac{1}{2 t}, \\
\left|c_{n}\right| & =\left|d_{n}\right| \leqq \min \left(\frac{1}{2 \pi|n|}, \frac{t}{2 \pi^{2} n^{2}}\right) .
\end{aligned}
$$

Proof. Use of the Fourier series

$$
\rho(x)=\sum_{n=-\infty}^{\infty} \frac{e(n x)}{i 2 \pi n}
$$

yields

$$
\begin{aligned}
t \int_{0}^{1 / t} \rho(x+u) d u & =\sum_{n=-\infty}^{\infty} c_{n} e(n x), \\
c_{n} & =t \frac{1-e\left(\frac{n}{t}\right)}{4 \pi^{2} n^{2}} .
\end{aligned}
$$

Thus

$$
\left|c_{n}\right|=\frac{t}{4 \pi^{2} n^{2}}\left|e\left(\frac{n}{t}\right)-1\right| \leqq \frac{t}{2 \pi^{2} n^{2}}
$$

From the identity

$$
e\left(\frac{n}{t}\right)-1=2 i e\left(\frac{n}{2 t}\right) \sin \frac{\pi n}{t}
$$

one has

$$
\left|e\left(\frac{n}{t}\right)-1\right| \leqq \frac{2 \pi|n|}{t} .
$$

Applying (36) to (33), one has

$$
\left|c_{n}\right| \leqq \frac{1}{2 \pi|n|}
$$


Similarly,

$$
\begin{aligned}
t \int_{-1 / t}^{0} \rho(x+u) d u & =\sum_{n=-\infty}^{\infty} d_{n} e(n x) \\
d_{n} & =t \frac{e\left(-\frac{n}{t}\right)-1}{4 \pi^{2} n^{2}} .
\end{aligned}
$$

Equations (33) and (38) show that

$$
\bar{d}_{n}=-c_{n}
$$

and, hence, that

$$
\left|d_{n}\right|=\left|c_{n}\right|
$$

Inequalities (34) and (37) are also valid for $d_{n}$; hence, the lemma is established.

\section{Lemma 4.}

$t>0, \quad y_{j} \quad$ real $\Rightarrow\left|\frac{1}{P} \sum_{j=1}^{P} \rho\left(y_{j}\right)\right| \leqq \frac{1}{P} \sum_{n=1}^{\infty} \min \left(\frac{1}{\pi n}, \frac{t}{\pi^{2} n^{2}}\right)\left|\sum_{j=1}^{P} e\left(n y_{j}\right)\right|+\frac{1}{2 t}$.

Proof. In the inequalities of Lemma $3, x$ is replaced by $y_{j}$ and summation is performed over $j$. Thus

$$
-\frac{1}{2} t+\frac{1}{P} \sum_{n=-\infty}^{\infty} d_{n} \sum_{j=1}^{P} e\left(n y_{j}\right) \leqq \frac{1}{P} \sum_{j=1}^{P} \rho\left(y_{j}\right) \leqq \frac{1}{P} \sum_{n=-\infty}^{\infty} c_{n} \sum_{j=1}^{P} e\left(n y_{j}\right)+\frac{1}{2 t}
$$

and, hence

$$
\begin{aligned}
-\frac{1}{2} t-\frac{1}{P} \sum_{n==\infty}^{\infty}\left|d_{n}\right|\left|\sum_{j=1}^{P} e\left(n y_{j}\right)\right| & \leqq\left|\frac{1}{P} \sum_{j=1}^{\infty} \rho\left(y_{j}\right)\right| \\
& \leqq \frac{1}{P} \sum_{n=-\infty}^{\infty}\left|c_{n}\right|\left|\sum_{j=1}^{P} e\left(n y_{j}\right)\right|+\frac{1}{2 t} .
\end{aligned}
$$

From Lemma 3, one has $\left|c_{n}\right|=\left|d_{n}\right|$; also, $\left|c_{n}\right|$ and $\left|\sum_{j=1}^{p} e\left(n y_{j}\right)\right|$ are even functions of $n$, hence,

$$
\left|\frac{1}{P} \sum_{j=1}^{P} \rho\left(y_{j}\right)\right| \leqq \frac{2}{P} \sum_{n=1}^{\infty}\left|c_{n}\right|\left|\sum_{j=1}^{P} e\left(n y_{j}\right)\right|+\frac{1}{2 t} .
$$

Use of the inequality

$$
\left|c_{n}\right| \leqq \min \left(\frac{1}{2 \pi n}, \frac{t}{2 \pi^{2} n^{2}}\right), \quad n>0,
$$

yields the lemma.

It will be convenient to introduce the function $\delta_{n, d}$ defined by

$$
\begin{aligned}
\delta_{n, d} & =1, & & d \mid n, \\
\delta_{n, d} & =0, & & d \nmid n .
\end{aligned}
$$

Lemma 5.

$x_{j}$ is defined by (26), $P=\phi(m) \Rightarrow\left|\sum_{j=1}^{P} e\left(n x_{j}\right)\right| \leqq \sum_{d \mid m} d \delta_{n, d}$. 
Proof. Since $\lambda$ is a primitive root modulo $m, \lambda^{j}$ runs through a reduced residue system. Let

$$
\lambda^{j} m x_{0} \equiv r \quad(\bmod m),
$$

where $r$ is the least nonnegative residue; then $r$ runs through a reduced residue system modulo $m$, and

$$
\sum_{j=1}^{P} e\left(n \lambda^{j} x_{0}\right)=\sum_{r} e\left(\frac{n r}{m}\right) .
$$

The sum $\sum_{r} e(n r / m)$ is a Ramanujan sum whose value can be expressed in terms of the Möbius function, $\mu(n)$ [2]. Let $c_{m}(n)$ denote the Ramanujan sum; then one has

$$
c_{m}(n)=\sum_{d \mid(m, n)} d \mu\left(\frac{m}{d}\right)
$$

Thus

$$
\left|c_{m}(n)\right| \leqq \sum_{d \mid(m, n)} d=\sum_{d \mid m} d \delta_{n, d} .
$$

It is now possible to establish

Theorem 5. $x_{j+1}=\left\{\lambda x_{j}\right\}, 0<x_{0}<1, m \geqq 3,\left(m x_{0}, m\right)=1, \lambda$ is a primitive root modulo $m, \omega(\alpha)$ is the distribution function, $P=\phi(m)$

$$
\Rightarrow|\omega(\alpha)-\alpha|<\frac{4}{\pi} \sqrt{\frac{3 \ln m}{P}} .
$$

Proof. Use of Lemmas 1 and 4 yields

$$
\left|\frac{1}{P} \sum_{j=1}^{P} \rho\left(x_{j}-\alpha\right)\right| \leqq \frac{1}{P} \sum_{n=1}^{\infty} \min \left(\frac{1}{\pi n}, \frac{t}{\pi^{2} n^{2}}\right)\left|\sum_{j=1}^{P} e\left(n x_{j}\right)\right|+\frac{1}{2 t},
$$

and

$$
|\omega(\alpha)-\alpha| \leqq \frac{2}{P} \sum_{n=1}^{\infty} \min \left(\frac{1}{\pi n}, \frac{t}{\pi^{2} n^{2}}\right)\left|\sum_{j=1}^{P} e\left(n x_{j}\right)\right|+\frac{1}{t}
$$

Lemma 5 is now used to provide an estimate for the trigonometric sum appearing in (50). Define the summation variable $\gamma$ by $n=\gamma d$, then

$$
|\omega(\alpha)-\alpha| \leqq \frac{2}{P} \sum_{d \mid m} \sum_{\gamma=1}^{\infty} \min \left(\frac{1}{\pi \gamma}, \frac{t}{\pi^{2} \gamma^{2} d}\right)+\frac{1}{t} .
$$

When

$$
t \geqq \pi d,
$$

one has

$$
\sum_{\gamma=1}^{\infty} \min \left(\frac{1}{\pi \gamma}, \frac{t}{\pi^{2} \gamma^{2} d}\right)=\sum_{1 \leqq \gamma \leqq t / \pi d} \frac{1}{\pi \gamma}+\sum_{\gamma>t / \pi d} \frac{t}{\pi^{2} \gamma^{2} d} .
$$

Since

$$
\sum_{1 \leqq \gamma \leqq t / \pi d} \frac{1}{\gamma}<1+\int_{1}^{[t / \pi d]} \frac{d x}{x} \leqq 1+\int_{1}^{t / \pi d} \frac{d x}{x}=1+\ln \frac{t}{\pi d}<\ln t,
$$


one has

$$
\sum_{1 \leqq \gamma \leqq t / \pi d} \frac{1}{\pi \gamma}<\frac{\ln t}{\pi}
$$

Similarly,

$$
\sum_{\gamma>t / \pi d} \frac{1}{\gamma^{2}}=\sum_{\gamma=[t / \pi d]+1} \frac{1}{\gamma^{2}}<\int_{[t / \pi d]}^{\infty} \frac{d x}{x^{2}}=\frac{1}{[t / \pi d]} .
$$

Since

$$
\frac{t}{\pi^{2} d[t / \pi d]} \leqq \frac{t}{\pi^{2} d}
$$

one has

$$
\sum_{\gamma>t / \pi d} \frac{t}{\pi^{2} \gamma^{2} d} \leqq \frac{t}{\pi^{2} d}
$$

and, hence, (53), (55), and (58) yield

$$
\sum_{\gamma=1}^{\infty} \min \left(\frac{1}{\pi \gamma}, \frac{t}{\pi^{2} \gamma^{2} d}\right)<\frac{\ln t}{\pi}+\frac{t}{\pi^{2} d}, \quad t \geqq \pi d .
$$

When

$$
t<\pi d
$$

one has

$$
\sum_{\gamma=1}^{\infty} \min \left(\frac{1}{\pi \gamma}, \frac{t}{\pi^{2} \gamma^{2} d}\right) \leqq \frac{t}{\pi^{2} d} \sum_{\gamma=1}^{\infty} \frac{1}{\gamma^{2}}<\frac{2 t}{\pi^{2} d} .
$$

Hence, using (59) and (61) in (51), one obtains

$$
|\omega(\alpha)-\alpha|<\frac{2}{P} \sum_{1 \leqq d \leqq t / \pi}\left(\frac{\ln t}{\pi}+\frac{t}{\pi^{2} d}\right)+\frac{2}{P} \sum_{1 \leqq d \leqq m} \frac{2 t}{\pi^{2} d}+\frac{1}{t} .
$$

Thus

$$
|\omega(\alpha)-\alpha|<\frac{4}{\pi^{2} P} t \ln t+\frac{4 t}{\pi^{2} P} \sum_{1 \leqq d \leqq m} \frac{1}{d}+\frac{1}{t} .
$$

In obtaining (63), the estimate of (54) was used. Since

$$
\sum_{1 \leqq d \leqq m} \frac{1}{d}<1+\ln m<2 \ln m, \quad m \geqq 3,
$$

one has

$$
|\omega(\alpha)-\alpha|<\frac{4 t \ln t+8 t \ln m}{\pi^{2} P}+\frac{1}{t} .
$$

Let

$$
0<t \leqq m
$$


then

$$
|\omega(\alpha)-\alpha|<\frac{12 \ln m}{\pi^{2} P} t+\frac{1}{t}
$$

The choice

$$
t=\frac{\pi}{2} \sqrt{\frac{P}{3 \ln m}}
$$

yields the inequality of the theorem.

When

$$
m=2^{\alpha}, \quad \alpha>2,
$$

the period is given by

$$
P=\frac{1}{2} \phi(m)=2^{\alpha-2},
$$

and there is no primitive root; hence, Theorem 5 is not directly applicable. The estimation of the trigonometric sum depended on Lemma 5 which requires $r$ to run over a reduced residue system. However, if one considers two distinct $\lambda$ 's, the powers of which together constitute a reduced residue system, then Lemma 5 is again operative and the estimate provided by Theorem 5 is valid. In fact, one need only consider the sequence obtained on setting $\lambda=5$ in order to obtain one half of the required reduced residue system; the other half is provided by the negatives of the first half.

Bell Telephone Laboratories, Inc.

Whippany, New Jersey

1. Joel N. Franklin, "Deterministic simulation of random processes," Math. Comp., v. 17,1963, pp. $28-59$. MR 26 *7125.

2. G. H. HARDY \& E. M. WRIGHT, An Introduction to the Theory of Numbers, 3rd ed., Clarendon Press, Oxford, 1954, pp. 234-239. MR 16, 673. 